\title{
Study of an Anisotropic Polymeric Cellular Material Under Compression Loading
}

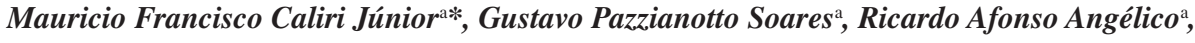 \\ Rodrigo Bresciani Canto ${ }^{\mathrm{b}}$, Volnei Tita ${ }^{\mathrm{a}}$ \\ ${ }^{a}$ Department of Aeronautical Engineering, Engineering School of São Carlos, \\ University of São Paulo - USP, Av. Trabalhador São-Carlense 400, São Carlos, SP, Brazil \\ ${ }^{b}$ Department of Materials Engineering, Federal University of São Carlos - UFSCar, \\ Rod. Washington Luís, Km 235, São Carlos, SP, Brazil
}

Received: April 7, 2011; Revised: February 7, 2012

\begin{abstract}
This paper emphasizes the influence of micro mechanisms of failure of a cellular material on its phenomenological response. Most of the applications of cellular materials comprise a compression loading. Thus, the study focuses on the influence of the anisotropy in the mechanical behavior of cellular material under cyclic compression loadings. For this study, a Digital Image Correlation (DIC) technique (named Correli) was applied, as well as SEM (Scanning Electron Microscopy) images were analyzed. The experimental results are discussed in detail for a closed-cell rigid poly (vinyl chloride) (PVC) foam, showing stress-strain curves in different directions and why the material can be assumed as transversely isotropic. Besides, the present paper shows elastic and plastic Poisson's ratios measured in different planes, explaining why the plastic Poisson's ratios approach to zero. Yield fronts created by the compression loadings in different directions and the influence of spring-back phenomenon on hardening curves are commented, also.
\end{abstract}

Keywords: polymeric foam, digital image correlation technique, compression tests

\section{Introduction}

In the literature, it is possible to find many contributions about cellular materials (e.g. foams), considering applications, experimental tests and different approaches for modeling ${ }^{1-5}$. To identify and determine the mechanical properties of cellular materials, standards and consolidated experimental methods developed for solid homogenous materials can be applied. However, for non homogenous materials (with high heterogeneity), some traditional methods, like strain measurements from strain gages, lead to the high variation in the obtained results. This can be explained through the weakening of the solid with the presence of the voids inside. In fact, when the material under investigation is a cellular structure like polymeric foams, the identification of the mechanical properties of this "material" is no longer straightforward. In addition, the polymeric foams are actually a micro structure with micro mechanisms of structural failure combined with responses from the base material, which is a polymer ${ }^{6-7}$. Therefore, the determination of the mechanical properties and strength values of cellular materials requires an extra attention when analyzing the results from standard test.

Regarding the challenge described above, some researchers ${ }^{8-10}$ decided to apply Digital Image Correlation (DIC) technique in order to obtain more accuracy results. Even though Digital Image Correlations techniques are well known these days, this paper seeks to expose the results of a specific DIC technique, named Correli, applied to rigid polymeric foam. In fact, Correli is used in order

*e-mail: voltitabr@yahoo.com.br to improve the understanding of the mechanical behavior and calculation of mechanical properties for polymeric cellular material under compression loadings. Also, the present work brings a lot more insights and results from the compression tests for closed-cell rigid poly (vinyl chloride) (PVC) foam. For example, the experimental results are discussed in detail, showing the anisotropy of the polymeric foam under compression and its influence on the stressstrain curves and on the determination of the mechanical properties. Besides, the present paper discusses not only about Poisson's ratio, but also about yield fronts created by the compression loading and the influence of spring-back phenomenon on hardening curves, using SEM (Scanning Electron Microscopy) analyses.

\section{Materials and Methods}

The closed-cell rigid foam named Divinycell ${ }^{\mathrm{TM}} \mathrm{H} 60$ from DIAB ${ }^{11}$ is adopted as the material to be investigated, whose base material is the poly (vinyl chloride) (PVC). This foam has a density of $60 \mathrm{~kg} \cdot \mathrm{m}^{-3}\left(\rho_{\text {rel }}=60 / 1400=0.043\right)$. It is important to mention that according to Gibson and Ashby ${ }^{1}$, cellular materials can be distinguished from porous materials through their relative density $\left(\rho_{\text {rel }}\right)$, which is the quotient of the cellular material density $(\rho)$ with the solid base one $\left(\rho_{\mathrm{s}}\right)$. For relative densities $\left(\rho_{\text {rel }}\right)$ below 0.3 , the material is classified as a cellular structure, otherwise the material is considered to be a porous material. Thus, in this work, the studied PVC foam can be classified as a cellular material. Within the manufacture's specifications, some 
mechanical properties are shown for the Divinycell ${ }^{\mathrm{TM}} \mathrm{H} 60$. In this paper, the material directions are chosen based on the plates' format as seen in Figure 1a. Thus the yield stress of 0.7-0.9 MPa and Young Modulus of 60-70 MPa shown by manufacturer datasheet corresponds to "out-of-plane" properties, i.e., properties in direction 3 (Figure 1a).

The test specimens are obtained from plates with dimensions $1200 \times 850 \times 40 \mathrm{~mm}$, discarding the material on the edges. Based on the ASTM D1621-04 $\mathrm{a}^{12}$ standard, five specimens were cut from the plate for the compression tests performed on the "out-of-plane" direction 3. Those specimens had $37.5 \mathrm{~mm}$ of height and a square section area of $40 \times 40 \mathrm{~mm}$. Other five specimens were cut from the plate for the compression tests performed on the in-plane direction 2. Those specimens had $35 \mathrm{~mm}$ of height and a square section area of $37 \times 37 \mathrm{~mm}$. After preliminary tests performed on the in-plane direction 1, Divinycell ${ }^{\mathrm{TM}} \mathrm{H} 60$ can be taken as transversally isotropic, because the response of material in this direction does not show significant difference compared to response in direction 2.

The tests were carried out in a universal test machine EMIC with a $10 \mathrm{kN}$ load cell. The specimens were investigated under cyclic tests with loading, unloading and reloading for different strain levels, which were obtained through a specific DIC technique, using a program named Correli ${ }^{\mathrm{Q} 4[13]}$. Figure $1 \mathrm{~b}$ shows an average texture in grayscale levels, used on the test specimens, required to employ the Correli (DIC technique). Due to the cellular nature of this PVC foam, the use of regular strain gages was not possible, because of the presence of very large voids. Also the high compression levels associated with the random size and disposal of cells can only be tracked with the aid of optical techniques. Even clip gages are very complicated to attach on the foam specimen.

The photographs were taken every 10 seconds with a CANON (EOS 50D) camera, using a lens with a $400 \mathrm{~mm}$ zoom and an aperture of f/4.5, ISO around 500 and a focus distance close to $1.5 \mathrm{~m}$. In addition, SEM (Scanning Electron Microscopy) analyses were performed on specimens by an equipment ZEISS model Leo440. The microcopies were carried out before and after the compression tests in order to investigate the microstructure of the polymeric foam specimens.

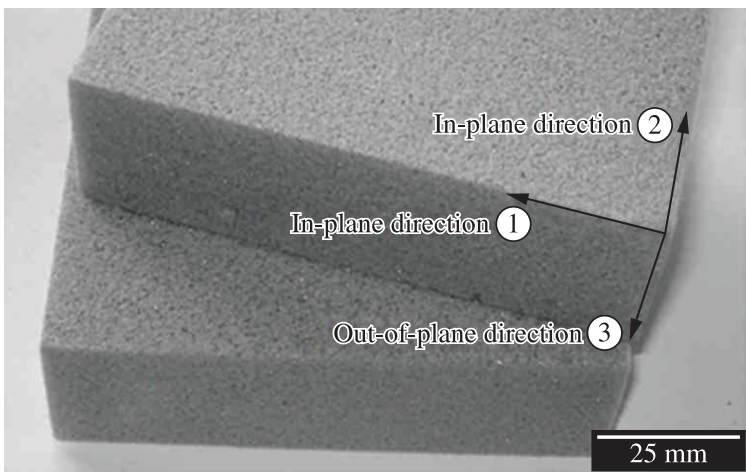

(a)

\section{Results and Discussion}

It is important to mention that the Figure 2 and Figure 3 represent the average behavior of a set of specimens for direction 3 and direction 2, respectively. All the compression test results were analyzed using the logarithmic strain $\left(\varepsilon_{\log }\right)$ and the associated Cauchy stress $\left(\sigma_{c}\right)$, due to the large strains involved. The average strains were obtained through regular calculations of logarithmic strains, $\left(\ln \left[1+\left(\Delta \mathrm{h} / \mathrm{h}_{0}\right)\right]\right.$, where $\mathrm{h}_{0}$ is the original height of the specimen) and the gradients of the displacements were calculated by the Correli ${ }^{\mathrm{Q} 4}$ program. Through the results, it can be seen that the crushing of the foam does not occur uniformly. The strain fields $\left(\varepsilon_{3}\right.$ and $\left.\varepsilon_{2}\right)$ for different levels (Figures 2 and 3) show that some "yield fronts" appear and propagate throughout the specimen.

For loadings on direction 3 (Figure 2), these fronts clearly delimit (up to a strain of approximately 0.85 ) the regions under only elastic strain (dark shades) or mainly plastic strain (clear shades). On the other hand, for directions 1 or 2 (Figure 3), the obtained results are somewhat different. Due to a greater structural homogeneity of the cellular material in plane 1-2, the strain fields do not exhibit well defined limits between the regions under mainly elastic or plastic strains when the material is under large strains. According to Gibson and Ashby ${ }^{1}$, the micro mechanisms of collapse of this PVC foam is not well defined due to its low relative density herein calculated $\left(\rho_{\text {rel }}=0.043\right)$. The formation of the "yield fronts" comprises the simultaneous initiation of plastic hinges and the micro buckling of the cellular structure, which made of the polymeric material.

Under strains up to $5 \%$, the material behaves in an elastic fashion. For strains over $5 \%$ up to $80 \%$ on the "out-of-plane" direction 3 (Figure 2), and up to $40 \%$ on the in-plane direction 2(1) (Figure 3), the material shows a nearly perfect plastic response, pointed by a yield stress of $0.76 \mathrm{MPa}$ and $0.40 \mathrm{MPa}$, respectively. One sees that on the in-plane direction 2(1) (Figure 3), there is a more evident hardening than on the "out-of-plane" direction 3. There is also a peak formation in Figure 3 referring to the maximum elastic stress of the material before the yield fronts begin to form and the material starts "yielding" with the propagation and diffusion of these yield fronts. Both phenomena stem

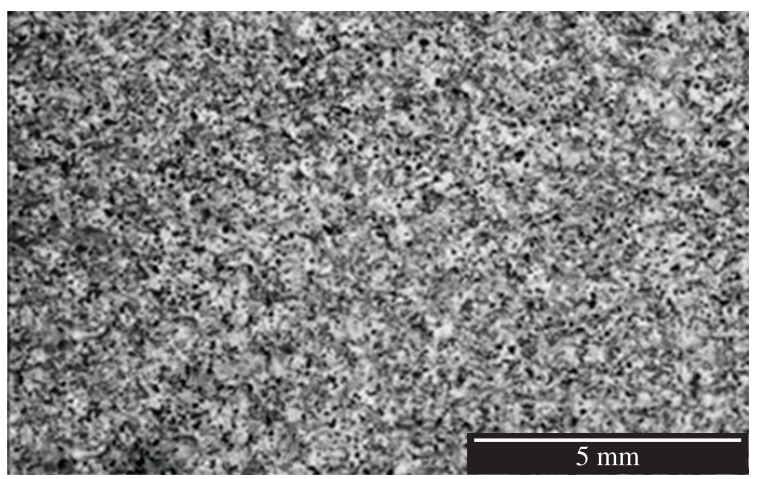

(b)

Figure 1. Divinycell H60 foam: a) Adopted index system and orthotropic planes; and b) Texture in grayscale levels applied to a test specimen for the DIC technique. 


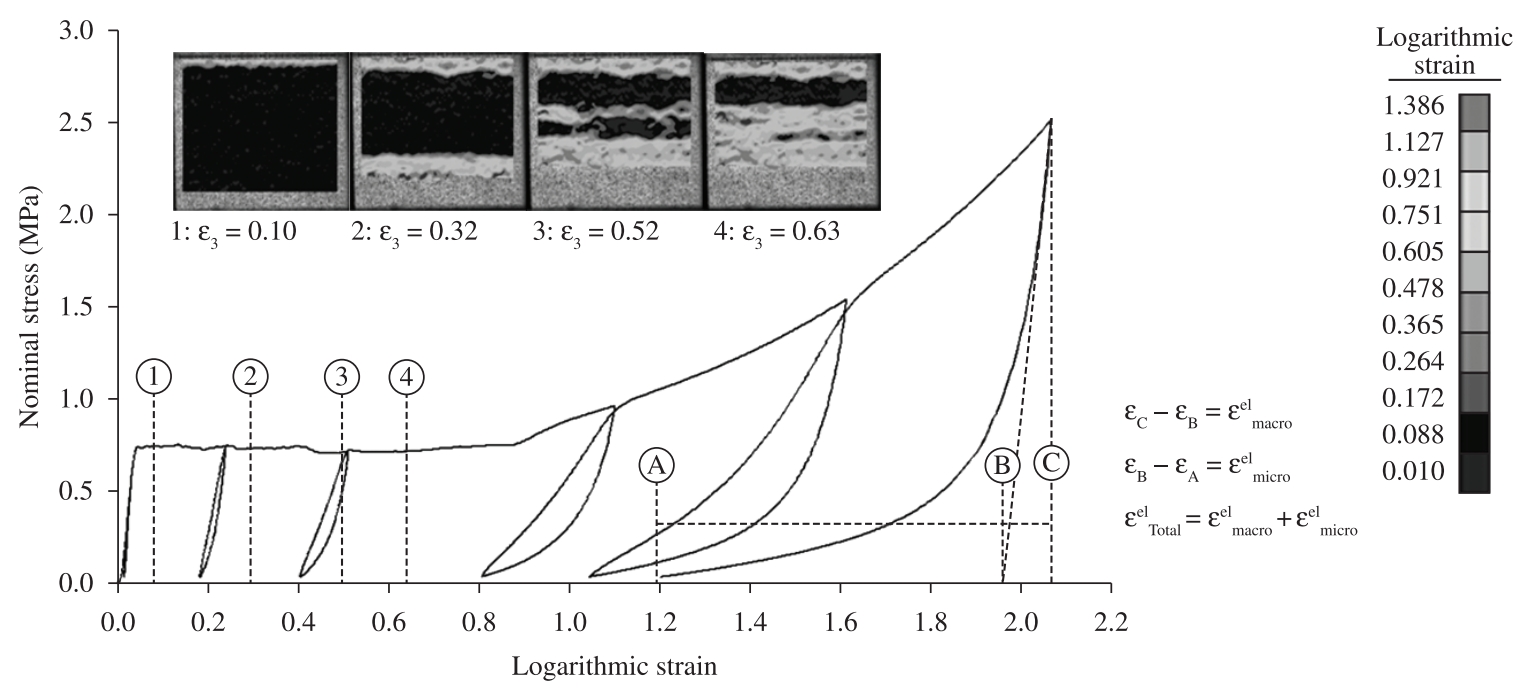

Figure 2. Uniaxial compression on direction 3 (curve via EMIC and gradient via Correli).

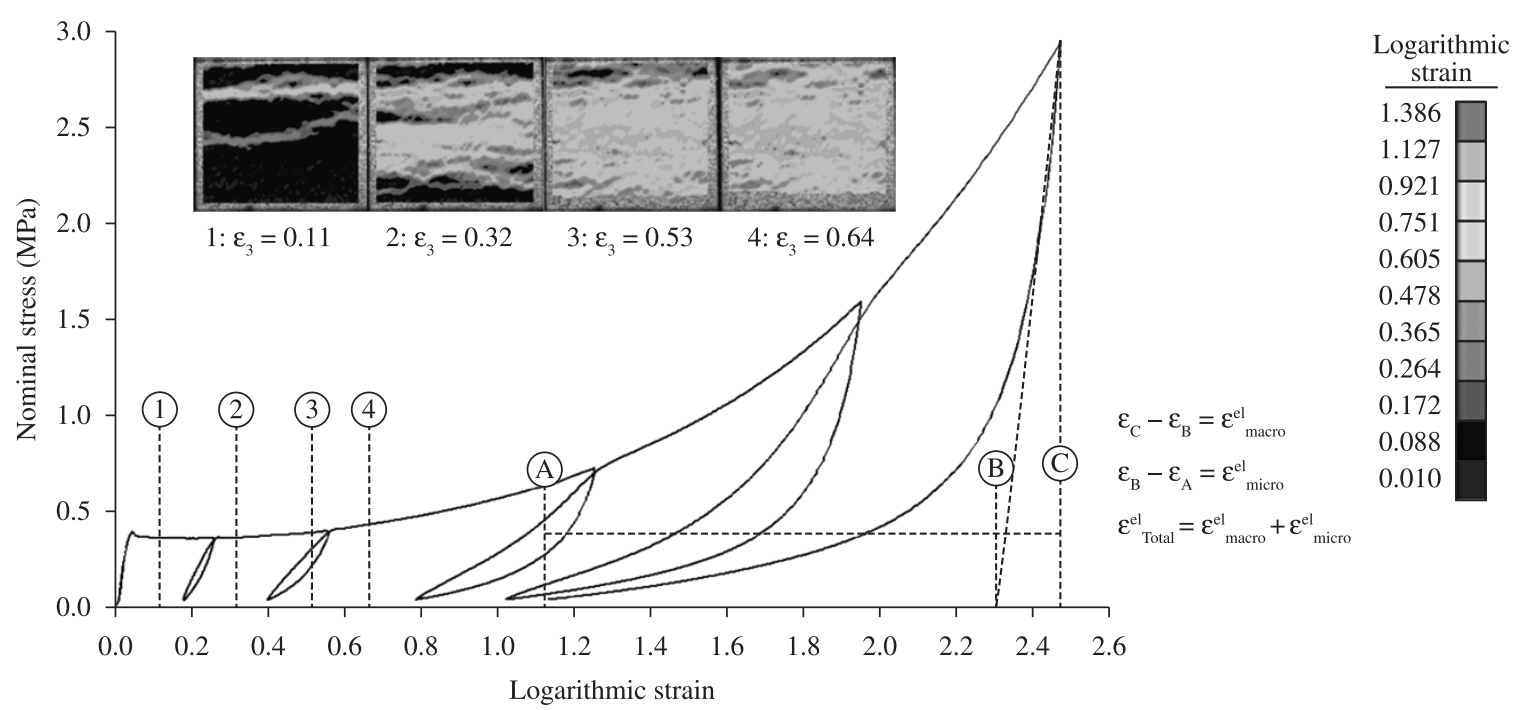

Figure 3. Uniaxial compression on direction 2 (1) (curve via EMIC and gradient via Correli).

from the greater homogeneity of the cellular structure in plane 1-2 (Figure 1a).

For strains greater than $50 \%$, regardless of the direction, the loops observed in the cycles of the compression curves suggest a phenomenological behavior of a viscous material. Also, as the foam crushes, its structure tends to a porous material reaching this limit for strain levels approximately above 1.9 for either direction. The pattern of these loops might be associated not only with the viscous behavior of the base solid material (PVC), but also, with the spring back behavior of the cells after micro buckling.

For the last unloading on stress-strain curves, it can be seen that the main failure mechanisms is the micro buckling. In Figures 2 and 3, it is observed that the total elastic strain $\left(\varepsilon_{\mathrm{C}}-\varepsilon_{\mathrm{A}}=\varepsilon^{\mathrm{el}}{ }_{\text {Total }}\right)$ comprises an elastic macro response $\left(\varepsilon_{\mathrm{C}}-\varepsilon_{\mathrm{B}}\right)$, and a portion related to the spring back of the buckled micro structure $\left(\varepsilon_{\mathrm{B}}-\varepsilon_{\mathrm{A}}\right)$. This influences the phenomenological hardening curves, because the total elastic strain for this cellular material is higher than expected for a regular continuum solid material. Thus, even though the actual permanent plastic strain might be evaluated experimentally, the inclusion of the spring back phenomenon in hardening curves is mandatory to correctly evaluate the strain energy of the foam. It is important to note that the hardening curves also depend on the loading direction. Therefore, in order to plot actual hardening curves, micro buckling elastic strains must be incorporated in the total inelastic strain, when the cellular material is under compression. This observation is essential to not underestimate the strain energy absorption capacity of the cellular material, especially when the hardening behavior is simulated by phenomenological material models.

The experimental results from the Correli were used to identify the Poisson' ratios of the PVC foam, also. Thus it 
was possible the identification of different Poisson's ratios for different strain levels of the material according to the anisotropy direction (Figure 4). The curves in Figure 4 exhibit a very similar behavior between the Poisson's ratios and such behavior is dependent on the anisotropy of the material, rendering two different sets of results. Therefore, well defined values for the elastic and plastic Poisson's ratios are extracted from both compression tests and the plastic Poisson's ratios tend to zero. This phenomenon is shown because the material shows collapse while occurs the packing of the cells filling out the empty spaces and keeping the section of the analyzed area nearly unchanged. Thus, for the main applications of these materials, which deal with large strain levels (like when absorbing high strain energy under impact compression load), it is usual to assume as zero the Poisson's ratio during the plastic regime analyses.

Table 1 shows a summarization of the results obtained from the compression tests. The Young Modulus obtained directly from the EMIC machine is lower than the one obtained from Correli, because the flexibility of the EMIC machine setup influences the results. Therefore, the properties determined by Correli should be considered more accuracy. Also, it is important to note that the material under investigation is indeed transversally isotropic and the Poisson's ratio from plane 3-1 is equal to that from plane 3-2.

In order to better understand the anisotropy and heterogeneity of the Divinycell H60 foam, it is necessary to know the basis of its manufacturing process ${ }^{14}$ and its final cellular structure features. The disposal of the cells can be seen in the micrographic images in Figure 5 for the planes 1-3 and 1-2. The image of plane 1-3, Figure 5a, shows bigger and more elongated cells, which correspond to the "out-of-plane" direction of the foam. In the image of Figure $5 b$ for the plane 1-2, one sees a geometric pattern of nearly circular and smaller cells. This particular arrangement and form of the cells was originated from the manufacturing process, which increases the number of irregular vertices in planes 1-2, as seen in Figure 5b. In addition, since more material accumulates in the vertices, the edges and faces in plane 1-2 are expected to be thicker. Consequently, such disposal of vertices in the micro structure increases the strength and stiffness of the "material" in the "out-of-plane" direction 3. Following the micro mechanical analysis involving square and rectangle frames proposed by

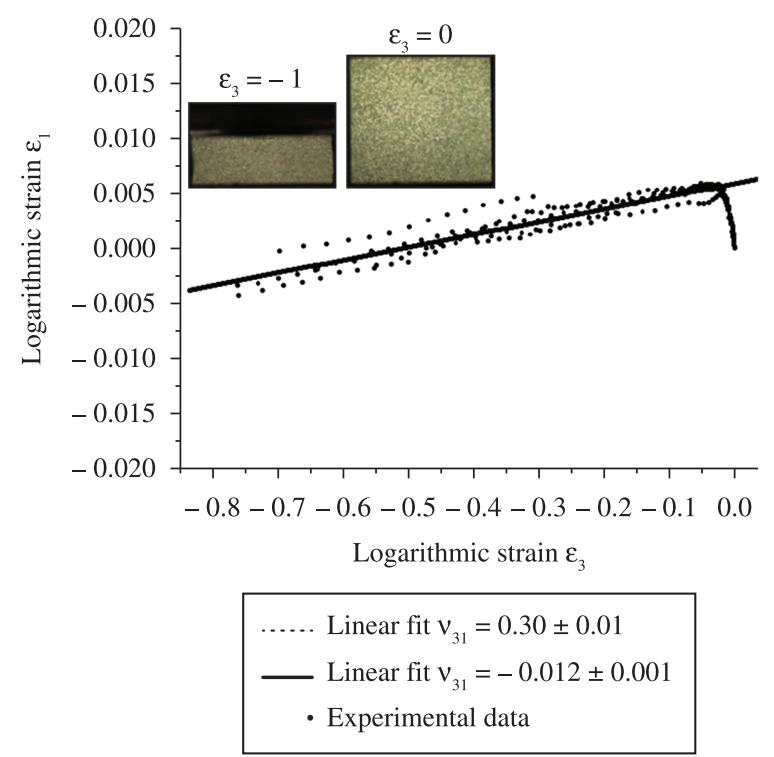

(a)

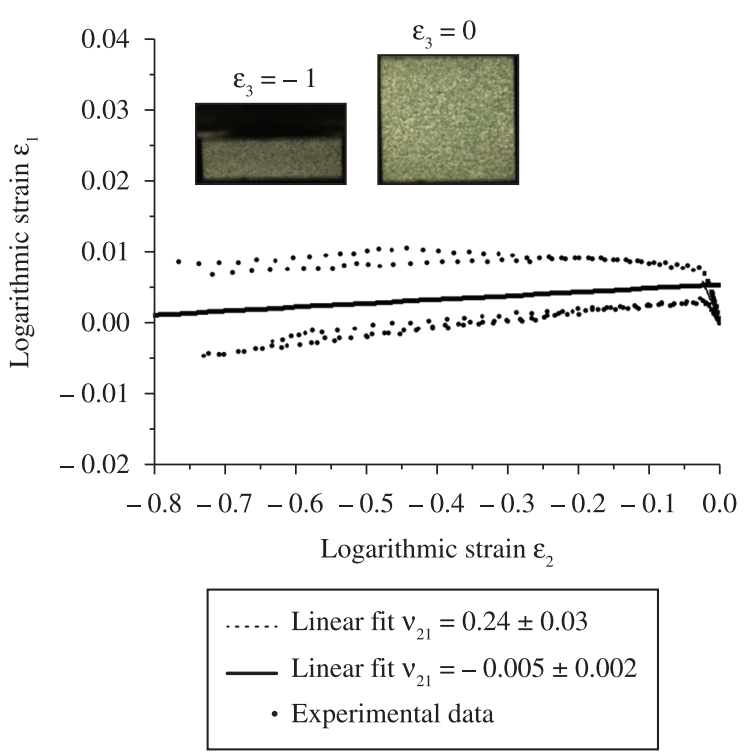

(b)

Figure 4. Poisson's ratio and logarithmic compression strain: a) plane 3-1; and b) plane 1-2.

Table 1. Mechanical properties and yield stresses of the polymeric foam Divinycell H60.

\begin{tabular}{ccccc}
\hline Property & Method & \multicolumn{3}{c}{ Compression test } \\
\cline { 3 - 5 } & & Direction (3) & Direction 1 (2) & $\Delta^{*}(\%)$ \\
\hline Young modulus, E $(\mathrm{MPa})$ & DIC & $53 \pm 6$ & $22 \pm 2$ & 58.5 \\
Yield stress, $\sigma_{\mathrm{y}}(\mathrm{MPa})$ & EMIC & $32 \pm 1$ & $16 \pm 3$ & 50.0 \\
Elastic poisson's ratio, $v_{\mathrm{el}}$ & EMIC & $0.76 \pm 0.02$ & $0.40 \pm 0.01$ & 47.9 \\
Plastic poisson's ratio, $v_{\mathrm{pl}}$ & DIC & $0.30 \pm 0.01$ & $0.24 \pm 0.03$ & 20.0 \\
\hline
\end{tabular}

\footnotetext{
$* \Delta=[$ Direction (3) - Direction (2)]/Direction (3).
} 


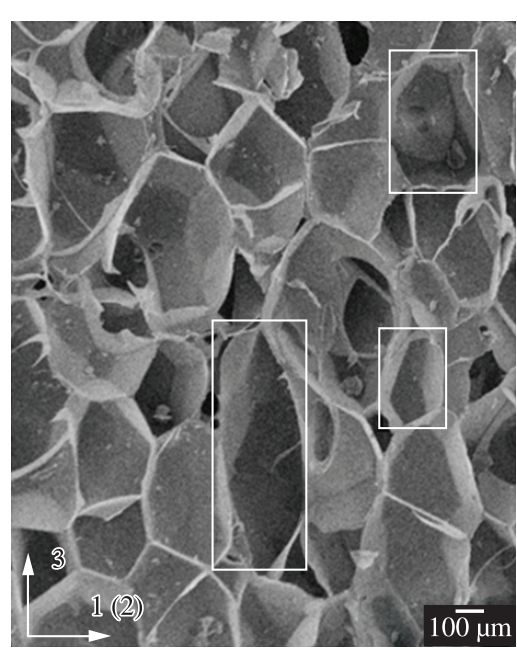

(a)

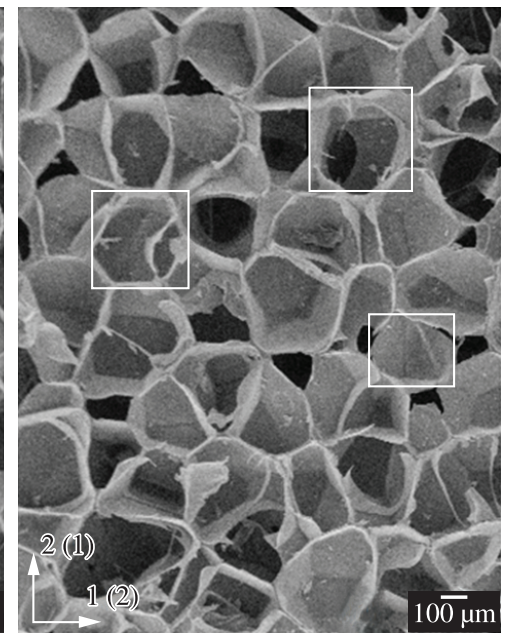

(b)

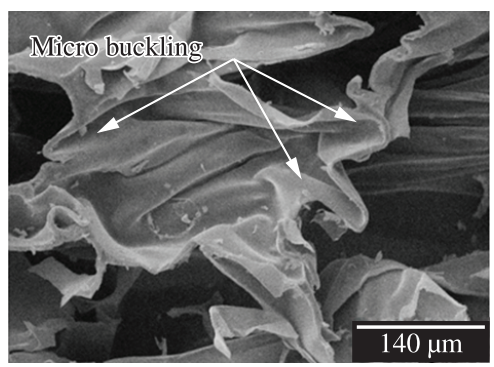

(c)

Figure 5. Micrographs of the H60 Divinycell foam: a) Morphology of plane 3-1(2); b) Morphology of plane 1-2; and c) Micro buckling of cells.

Gibson and Ashby ${ }^{1}$, it is possible to analytically obtain the mechanical properties of this cellular structure. However, such approach requires an extra statistical treatment of images which was not the focus of this article. Finally, in Figure 5c, a specimen of crushed and completely relaxed material (after the spring back phenomenon) was examined via SEM and the failure mechanisms of the material were analyzed. In this micrograph, the failure mechanisms of micro buckling of faces and edges and the formation of plastic hinges can be confirmed.

\section{Conclusions}

The heterogeneity and anisotropy have a great influence in the studied material (closed-cell rigid poly (vinyl chloride) (PVC) foam) response under different compression loadings directions. For example, the yield stress in the direction 3 is almost twice in the direction 2 .

The use of Correli (DIC technique) was very strategy in order to determine the mechanical properties of the PVC cellular material, as well as to observe the yield fronts due to its micro structure behavior. Besides, it was not possible to use clip gages and strain gages due to the high heterogeneity of this cellular material and its failure mechanisms, especially under large compression displacements. Also, the DIC technique was of particular assistance at this stage, since it is an optic technique and free of parasitic strains.
This can be confirmed by the Young Modulus calculation, because the result of Correli is about 1.66 times of EMIC measured directly. Therefore, considering cellular materials, foams, and similar materials, the use of an optical technique is highly recommended.

The failure mechanisms of the micro structures directly influence the plastic Poisson's ratios, which are close to zero in both directions ( 3 and 2). The results from Correli and SEM micrographs supported and explained how the collapse with the packing of the cells filling out the empty spaces. Besides, failure mechanisms influence the hardening curves due to the spring-back phenomenon, observed in stress-strain curves and in SEM micrographs. Therefore, Poisson's ratios and hardening curves must be set with caution in project calculations of components made of PVC foams.

Future works can focus on the phenomenological rate-dependent behavior under large strains, being such strains composed of micro and macro mechanical contributions.

\section{Acknowledgements}

The authors would like to thank the Sao Paulo Research Foundation (FAPESP - process number: 2009/00544-5) for the financial support. The authors would also like to thank National Council for Research and Development (CNPq).

\section{References}

1. Gibson LJ and Ashby M. Cellular solids: structures \& properties. England: Pergamon; 1988.

2. Abrate S. Criteria for yielding or failure of cellular materials. Journal of Sandwich Structures and Materials. 2008; 10:5-51. http://dx.doi.org/10.1177/1099636207070997

3. Deshpande VS and Fleck NA. Multi-axial yield behavior of polymer foams. Acta materialia. 2001; 49: 1859-66. http:// dx.doi.org/10.1016/S1359-6454(01)00058-1

4. Ragab AR and Saleh CAR. Evaluation of constitutive models for voided solids. International Journal of Plasticity. 1999; 15:1041-65. http://dx.doi.org/10.1016/ S0749-6419(99)00024-8

5. Viana GM and Carlsson LA. Mechanical properties and fracture characterization of cross-linked PVC foams. Journal of Sandwich Structures and Materials. 2002; 4:99-103. http:// dx.doi.org/10.1177/1099636202004002227 
6. Gong L, Kyriakides S and Jang W-Y. Compressive response of open-cell foams. Part I: Morphology and elastic properties. International Journal of Solids and Structures. 2005; 42:135579. http://dx.doi.org/10.1016/j.ijsolstr.2004.07.023

7. Gong L and Kyriakides S. Compressive response of open cell foams Part II: Initiation and evolution of crushing. International Journal of Solids and Structures. 2005; 42:1381-99. http:// dx.doi.org/10.1016/j.jijsolstr.2004.07.024

8. Grédiac M, Toussaint E and Pierron F. Special virtual fields for the direct determination of material parameters with the virtual fields method. 1- Principle and definition. International Journal of Solids and Structures. 2002; 39:2691-2709. http:// dx.doi.org/10.1016/S0020-7683(02)00127-0

9. Grédiac M, Toussaint E and Pierron F. Special virtual fields for the direct determination of material parameters with the virtual fields method. 2-Application to in-plane properties. International Journal of Solids and Structures. 2002; 39:2707-30. http:// dx.doi.org/10.1016/S0020-7683(02)00128-2
10. Pierron F. Mechanical properties of low density polymeric foams obtained from full-field measurements. EPJ Web of Conferences. 2010; 6:37006. http://dx.doi.org/10.1051/ epjconf/20100637006

11. Diab. Technical Manual: Divinycell H. Available from: <http:// www.diabgroup.com/europe/literature/e_lit_man.html $>$. Access in: 02/11/2010.

12. American Society for Testing and Materials - ASTM. ASTM D 1621-04a: Standard Test Method for Compressive Properties of Rigid Cellular Plastics. ASTM; 2004.

13. Besnard G,HildF and Roux S. "Finite-Element" displacement fields analysis from digital images: Application to Portevin-Le Châtelier bands. Experimental Mechanics. 2006; 46: 789. http://dx.doi. org/10.1007/s11340-006-9824-8

14. Vilar W. Química e Tecnologia dos Poliuretanos. Available from: <http://www.poliuretanos.net/>. Access in: 05/01/2010. 